\title{
Estudio comparativo de la calidad de la canal y la carne de lechales de raza Churra Tensina y dos tipos ovinos comerciales amparados bajo marcas de calidad
}

\author{
Guerrero, A. ${ }^{\circledR}$; Campo, M.M.; Olleta, J.L.; Resconi, V.C.; Muela, E. y Sañudo, C.
}

Departamento de Producción Animal y Ciencia de los Alimentos. Universidad de Zaragoza. Zaragoza. España.

\section{Palabras ClaVE ADICIONALES}

Composición tisular.

Consumidores.

Lechal.

Perfil sensorial.

Textura.
Ácidos grasos.

\section{RESUMEN}

Se estudió la calidad de la canal y la carne de lechales ovinos comerciales de raza Churra Tensina $(n=26)$ según la época de cría y sacrificio (primavera vs. otoño). Estos corderos se compararon con otros tipos comerciales amparados bajo marcas de calidad reconocidas en el mercado con denominación de Indicación Geográfica Protegida (IGP): Lechazo de Castilla-León ( $n=13$ ) y Ternasco de Aragón ( $n=13)$. Todos los corderos fueron criados, seleccionados y sacrificados en condiciones comerciales. Tras el sacrificio, se valoró la calidad de la canal y de la carne en el músculo Longissimus thoracis y lumborum (LTL). Los lechales de Churra Tensina presentaron diferencias según la época de producción, fundamentalmente en las variables relacionadas con la composición tisular de la canal, presentando, los lechales de primavera, un mayor porcentaje de músculo y hueso, y menor de grasa que los de otoño ( $\leq \leq 0,001)$, así como una mayor proporción de los ácidos grasos C 12:0, CLA, C 20:2n-3 o C 22:0 ( $\leq \leq 0,001)$ en la grasa intramuscular. Estas diferencias de composición no se reflejaron en el análisis sensorial, ya sea mediante panel entrenado o consumidores. La carne de los lechales de la raza Churra Tensina presentó una terneza superior $(p \leq 0,001)$ a la de los productos IGP con los que se comparó, tanto instrumentalmente como sensorialmente (panel entrenado) y una mayor aceptabilidad tanto general, como del sabor y de la terneza (consumidores) ( $p \leq 0,001)$. Los resultados obtenidos reflejarían el potencial de dicha raza para la producción de lechales de calidad, en ambas estaciones del año.

\section{Comparative study of carcass and meat quality from suckling Churra Tensina breed and two commercial lambs recognized with quality brands}

\section{SUMMARY}

\section{ADDITIONAL KEYWORDS}

\section{Consumer test.}

Fatty acids.

Sensory profile.

Suckling lambs.

Texture.

Tissular composition.

\section{INFORMACIÓN}

\section{Cronología del artículo.}

Recibido/Received: 11.12.2014

Aceptado/Accepted: 1.6.2015

On-line: 16.9.2015

Correspondencia a los autores/Contact e-mail:

guerrerobarrado@gmail.com
The aim of this study was to evaluate the effect of the rearing and slaughtering season (spring vs. autumn) on the carcass and meat quality of suckling lambs from Churra Tensina breed $(n=26)$, and to compared the results with two commercial lambs within Protected Geographical Indication (PGI) Lechazo de Castilla-León $(n=13)$ and Ternasco de Aragón $(n=13)$. All animals were reared, selected and slaughtered under commercial conditions. After slaughter, carcass and meat quality were evaluated in the Longissimus thoracis lumborum (LTL) muscle. Slaughter season mainly affected both the tissue composition of the carcass, presenting Churra Tensina breed suckling lambs from spring greater muscle and bone, and lower fat percentages $(p \leq 0.001)$ and a higher proportion of fatty acids: C 12:0, CLA, C $20: 2 n-3$ and C 22:0 (p $\leq 0.001)$ in IMF. However, those differences in quality were not reflected when sensory methodologies (trained panel or consumers analyses) were conducted. Related to commercial lambs from the GPI's studied, meat from Churra Tensina breed showed a higher tenderness ( $\leq 0.001$ ) evaluated by instrumental and sensory (trained panel), as well as a higher scores in overall, flavour or tenderness acceptabilities evaluated by consumers ( $p \leq 0.001)$. Results would indicate a good potential of the Churra Tensina breed to produce high quality suckling lambs, in both seasons of the year.

\section{INTRODUCCIÓN}

La Churra Tensina es una raza ovina autóctona aragonesa registrada en el Catálogo Oficial de Razas de Ganado de España dentro del grupo de razas en peligro de extinción. Dicha raza pertenece al tronco Churro, Ovis aries studeri, cuyas cualidades genéticas y productivas se encuentran claramente diferenciadas del resto de razas de Aragón (Calvo et al., 2011; Panea et al., 2013). La mayoría de sus ganaderías se localizan 
en el Pirineo Central, en las comarcas del Alto Gallego y Sobrarbe, permitiendo la utilización de unos recursos naturales de forma no competitiva con otras especies, y transformándolos en productos que potencialmente pueden tener un elevado interés gastronómico en la región, la cual cuenta con un elevado interés turístico.

La raza Churra Tensina tiene una alta capacidad de adaptación y presenta adecuados rendimientos en diferentes sistemas de manejo y alimentación, ya sea en estabulación o pastoreo (Joy et al., 2008). En relación a los tipos comerciales existentes en dicha raza, estos son variados (lechal, ternasco, cordero o pastero) como se ha documentado en estudios anteriores (Olleta et al., 1992; Sanz et al., 2008). Por otra parte y a pesar de que diversos trabajos han mostrado la viabilidad y versatilidad de la raza en diferentes sistemas productivos, la información disponible sobre la misma todavía podría considerarse escasa, especialmente si se compara con la existente en otras razas ovinas españolas y muy especialmente con productos ovinos de alto reconocimiento como son el Lechazo de Castilla-León y el Ternasco de Aragón, ambos amparadas por sus respectivas Indicaciones Geográficas Protegidas (IGP).

Por otra parte, el desarrollo de marcas de calidad en carne fresca y la demanda creciente por parte del consumidor de productos locales reclaman la existencia de estudios en los que se tipifiquen dichos productos y se analice su variabilidad y calidad en comparación con otros ya consolidados en el mercado de carne (Martínez-Cerezo et al., 2005a). En esta línea, los estudios en los que se comparan diversos productos comerciales pueden dar una visión más realista del mercado y complementar, desde otro punto de vista, otros estudios más experimentales. El tipo comercial lechal es una opción productiva de creciente interés en aquellas explotaciones con marcada estacionalidad de los recursos pastorales, ya que los costes de alimentación se ven reducidos y permite una diversificación de los productos (Sanz et al., 2008; Panea et al., 2010). Además, la cría de estos animales y su posterior consumo tienen todavía un marcado carácter temporal, antiguamente asociado a la propia estacionalidad reproductiva de los ovinos, estando ligado a determinadas épocas en las que la demanda aumenta debido a tradiciones socioculturales o religiosas (otoño-invierno: Navidades y turismo invernal; primavera: Pascua). Durante estos periodos, las condiciones de cría cambian debido a la diferente disponibilidad de los recursos existentes, por lo que consecuentemente podrían variar algunas características de su calidad (Mazzone et al., 2010; D’Alessandro et al., 2013).

Por todo ello y debido a las posibilidades que podría tener la raza Churra Tensina en abastecer los centros de consumo locales (carnicerías y restaurantes) incrementando el valor añadido de sus productos, se ha realizado este trabajo, cuyo objetivo fundamental, además de tipificar las características de lechales comerciales de dicha raza, ha sido comparar su calidad con la de otros productos ya posicionados en el mercado español y reconocidos con marcas dentro del marco europeo, como son el Lechazo de Castilla-León y el Ternasco de Aragón.

\section{MATERIAL Y MÉTODOS}

\section{ANIMALES Y DISEÑO EXPERIMENTAL}

Se utilizaron un total de 52 animales, todos ellos machos enteros, pertenecientes a las razas Churra Tensina $(n=26)$, Churra $(n=13)$ y Rasa Aragonesa $(n=13)$, estando esos dos últimos grupos amparados bajo las Indicaciones Geográficas Protegidas (IGP) Lechazo de Castilla-León y Ternasco de Aragón, respectivamente. Los animales de raza Churra Tensina pertenecían a la categoría comercial lechal, cuyas características de producción se detallan en Sanz et al. (2008). Los lechales, seleccionados directamente por la Asociación de Ganaderos bajo un estricto criterio de representatividad del producto, fueron alimentados exclusivamente con leche materna, y sacrificados en dos lotes separados en el tiempo: trece lechales nacidos y sacrificados en primavera. Para este grupo, las madres pasaron el invierno estabuladas, con alimentación a base de heno de prado o de alfalfa y un suplemento a base de pienso, incrementando esta suplementación en el último mes de gestación y lactancia. Y los restantes trece lechales nacidos y sacrificados en otoño, grupo para el cual las madres pasaron el verano en puertos de alta montaña del Pirineo Central, de donde se recogieron unos 20 días antes del parto. Una vez en la explotación salieron a pastar en praderas del valle hasta el parto, momento en el cual fueron estabuladas con alimentación basada en heno de prado o de alfalfa, paja y pienso compuesto. Los animales de IGP fueron criados bajo las condiciones comerciales, exigidas y recogidas en los respectivos pliegos de condiciones (BOE, 2011; MAGRAMA, 2011), siendo seleccionados por el propio personal técnico de los Grupos Cooperativos COLEAR con base en Aranda de Duero (Lechazo de Castilla-León) y Pastores de Aragón con base en Zaragoza (Ternasco de Aragón), dentro de los animales que normalmente comercializan al amparo de sus marcas de calidad.

Los sacrificios se realizaron en mataderos comerciales, siguiendo la normativa sobre protección de animales en el momento del sacrificio. El de los animales de raza Churra Tensina se realizó en Sabiñánigo (Huesca), el del Lechazo de Castilla-León en Aranda de Duero (Burgos) y el del Ternasco de Aragón en Mercazaragoza (Zaragoza). Todos los animales de un mismo tipo comercial fueron sacrificados en un mismo día.

\section{MUESTREO Y ANÁLISIS LABORATORIALES}

A las 24 horas post-sacrificio todas las canales fueron transportadas en condiciones de refrigeración al laboratorio de la Facultad de Veterinaria de Zaragoza, donde se obtuvo el peso canal fría (PCF). Allí se evaluó el pH en el Longissimus lumborum por medio de un electrodo de penetración.

De cada canal se retiró la espalda izquierda, siguiendo la metodología descrita por Colomer et al. (1988). Éstas se envasaron al vacío y se conservaron en congelación $\left(-18^{\circ} \mathrm{C}\right)$ hasta su disección, con la que se evaluó la composición porcentual de músculo, grasa subcutánea, grasas intermuscular, hueso y otros (grandes vasos, tendones y el ganglio pre-escapular). 
Además de la espalda, se extrajeron ambos músculos Longissimus thoracis y lumborum (LTL), utilizándose posteriormente la porción torácica del LTL izquierdo para el análisis de ácidos grasos (T3-T11) y el resto (T11-L6) para análisis sensorial mediante panel entrenado. La porción torácica del LTL derecho se destinó para los análisis de textura y la lumbar, para realizar el estudio con consumidores. Todas las muestras fueron envasadas al vacío a las $24 \mathrm{~h}$ post-mortem. La muestra de ácidos grasos se congeló inmediatamente, y el resto de muestras fueron refrigeradas a $4^{\circ} \mathrm{C}$ hasta que completaron el tiempo de maduración post-sacrificio de 4 días, momento en que fueron congeladas $\left(-18^{\circ} \mathrm{C}\right)$ hasta su posterior análisis.

La textura se valoró mediante la utilización del equipo universal Instron 4301 (Instron Limited Corporation, High Wycombe, United Kingdom) y la célula de Warner Bratzler. Para ello, la carne se calentó, envasada al vacío, en baño maría $\left(75^{\circ} \mathrm{C}\right)$ hasta que las muestras alcanzaron una temperatura interna de $70^{\circ} \mathrm{C}$, monitorizada por un termómetro Jenway 2000 dotado con una sonda de penetración. Posteriormente, cada muestra fue dividida en prismas de $1 \mathrm{~cm} \times 1 \mathrm{~cm}$ de sección, evaluándose la fuerza necesaria para cortar totalmente la muestra (Campo et al., 2000), expresándose los resultados como dureza de la carne $\left(\mathrm{kg} / \mathrm{cm}^{2}\right)$ y carga máxima $(\mathrm{kg})$.

El color de la carne se midió a nivel de T11 sobre la superficie del LTL, tras 30 minutos de oxigenación. Se utilizó un espectrofotómetro Minolta CM 2002 (Konika Minolta Inc., Tokyo, Japan) con un iluminante D65 y $10^{\circ}$ como observador estándar, siguiendo el sistema CIE L*a*b* (CIE, 1976) realizándose tres medidas en cada muestra. Las variables analizadas fueron luminosidad $\left(\mathrm{L}^{*}\right)$, índice de rojo $\left(\mathrm{a}^{*}\right)$ e índice de amarillo $\left(\mathrm{b}^{*}\right)$.

La extracción de la grasa intramuscular se realizó tras la descongelación de las muestras (Bligh y Dyer, 1959), seguido de una metilación con KOH en metanol. La identificación de los ácidos grasos se realizó mediante cromatografía de gases, utilizando un cromatógrafo HP 6890 de columna capilar SP 2380 (100 m x $0.25 \mathrm{~mm} \times 0.20 \mu \mathrm{m})$, un FID (Flame Ionization Detector) como detector y nitrógeno como gas portador (Carrillho et al., 2009).

El análisis sensorial con panel entrenado se realizó según las normas UNE-EN ISO 8589 (2010), utilizando cabinas normalizadas con luz roja. El panel constaba de nueve personas seleccionadas y entrenadas para realizar dichas evaluaciones (UNE-EN ISO 8586-2, 2009). Las muestras se descongelaron en refrigeración durante 24 horas y posteriormente fueron cocinadas en un grill de doble placa precalentado a $200^{\circ} \mathrm{C}$, sin aditivos, hasta alcanzar una temperatura interna de $70^{\circ} \mathrm{C}$, la cual se controló mediante termómetro con sonda de penetración. Una vez que las muestras fueron cortadas $(2 \times 2 \mathrm{~cm})$, etiquetadas y distribuidas, los panelistas evaluaron en cada una de ellas diez atributos (olor a cordero, olor a grasa, olor lácteo, terneza, jugosidad, fibrosidad, flavor a cordero, flavor a grasa, flavor lácteo y flavor metálico), mediante una escala semi-estructurada de $10 \mathrm{~cm}$ desde nada intenso, tierno, jugoso o fibroso (según atributo) a extremadamente intenso, tierno, jugoso o fibroso respectivamente. Estos descriptores fueron decididos por el propio panel en una sesión previa de entrenamiento con carne equivalente a la que posteriormente se utilizara en el estudio. El diseño fue equilibrado, constando de 4 sesiones, 4 platos por sesión y tres muestras por plato.

El análisis de consumidores se realizó bajo condiciones controladas en sala con luz blanca, estando dividido en 12 sesiones, en total participaron 120 personas, siendo el 51,7\% hombres y el 48,3\% de mujeres; repartidos en los siguientes intervalos de edad: un 28,6\% tenía menos de 25 años, el 15,1 \% entre 26 y 40, el 31,1\% entre 41 y 55, y el 25,2\% tenía más de 55 años. Para la preparación culinaria de las muestras se siguió el procedimiento descrito en análisis con panel. Cada consumidor evaluó una muestra de cada uno de los cuatro lotes experimentales. Para evitar posibles efectos de unas muestras sobre otras, éstas se evaluaron en distinto orden para cada consumidor (Macfie et al., 1989), siguiendo un diseño de bloques equilibrados. Se evaluó en cada muestra la aceptabilidad de la terneza y del sabor, así como su aceptación general, mediante una escala hedónica estructurada de 9 puntos, que variaba desde 1 (me desagrada extremadamente) a 9 (me gusta extremadamente), en la que se eliminó el punto medio 5 (ni me gusta ni me desagrada) de acuerdo con la metodología descrita por Font i Furnols et al., 2008.

\section{ANÁLISIS ESTADÍSTICO}

Se utilizó el paquete estadístico SPSS v19.0 para estudiar el efecto del tipo comercial (Churra Tensina primavera, Churra Tensina otoño, Lechazo de CastillaLeón y Ternasco de Aragón) sobre las variables descritas mediante un análisis de varianza (GLM) considerando el tipo comercial como efecto fijo.

En el análisis sensorial, un primer GLM fue realizado con el panelista como efecto fijo, además de la sesión y del tipo de cordero, para analizar el consenso en las valoraciones. Posteriormente, se analizó el efecto tipo de cordero en un segundo análisis además del plato (sesión). En el test de consumidores, además del tipo de cordero como efecto fijo, se consideró al consumidor como aleatorio.

Cuando el efecto tipo de cordero fue significativo, las diferencias entre medias se evaluaron mediante el test de Duncan $(p \leq 0,05)$. Los resultados se presentan como medias de cada grupo, así como con el error estándar de la media.

\section{RESULTADOS Y DISCUSIÓN}

\section{Calidad de la canal}

\section{PESO Y COMPOSICIÓN TISULAR}

Los lechales de la raza Churra Tensina fueron los más ligeros dentro de los cuatro tipos de canales consideradas en el trabajo, posiblemente por estrictas razones de tipo comercial. Las canales de los lechales de primavera fueron más ligeras que las de otoño (tabla I) lo que se podría deber a: razones de tipo comercial, a posibles diferencias en la edad de sacrificio y a diferencias provocadas por el propio sistema de producción. Así, en referencia a este último efecto, Mazzone et al. 
Tabla I. Peso canal, composición tisular, textura y color de lechales comerciales de raza Churra Tensina sacrificados en primavera $u$ otoño respecto a dos tipos ovinos con Indicación Geográfica Protegida (IGP) (Carcass weight, tissular composition, texture analyses and color of Churra Tensina commercial suckling lambs slaughtered in spring or autumn, compared with two commercial lambs within a Protected Geographical Indication (IGP)).

\begin{tabular}{|c|c|c|c|c|c|c|}
\hline & Churra tensina primavera & Churra tensina otoño & LechazoํIGP & T.A. ${ }^{2}$ IGP & e.e.m & Sig. \\
\hline PCF & $4,66^{d}$ & $5,43^{c}$ & $5,85^{\mathrm{b}}$ & $9,80^{\mathrm{a}}$ & 0,29 & $* * *$ \\
\hline \multicolumn{7}{|l|}{ COMPOSICIÓN TISULAR } \\
\hline \% Músculo & $59,02^{c}$ & $57,19^{d}$ & $60,60^{\mathrm{b}}$ & $62,57^{\mathrm{a}}$ & 0,37 & *** \\
\hline$\%$ Grasa subcutánea & $4,54^{\mathrm{b}}$ & $6,19^{a}$ & $2,81^{\mathrm{c}}$ & $4,19^{b}$ & 0,24 & $* * *$ \\
\hline$\%$ Grasa intermuscular & $8,98^{b}$ & $10,04^{a}$ & $6,71^{d}$ & $7,72^{\mathrm{c}}$ & 0,24 & *** \\
\hline$\%$ Grasa total & $13,52^{\mathrm{b}}$ & $16,23^{\mathrm{a}}$ & $9,53^{c}$ & $11,91^{b}$ & 0,46 & *** \\
\hline$\%$ Hueso & $24,00^{\mathrm{b}}$ & $22,81^{c}$ & $25,51^{a}$ & $22,12^{c}$ & 0,27 & $* * *$ \\
\hline$\%$ Otros & $3,45^{b}$ & $3,77^{\mathrm{b}}$ & $4,36^{a}$ & $3,40^{\mathrm{b}}$ & 0,11 & ** \\
\hline Músculo/hueso & $2,47^{\mathrm{b}}$ & $2,51^{b}$ & $2,38^{b}$ & $2,85^{a}$ & 0,03 & $* * *$ \\
\hline $\mathrm{PH}$ & $5,69^{a}$ & $5,59^{b}$ & $5,70^{\mathrm{a}}$ & $5,72^{\mathrm{a}}$ & 0,01 & $* * *$ \\
\hline \multicolumn{7}{|l|}{ TEXTURA } \\
\hline Carga máxima-WB (kg) & $2,15^{b}$ & $2,21^{\mathrm{b}}$ & $2,71^{\mathrm{b}}$ & $4,38^{\mathrm{a}}$ & 0,18 & *** \\
\hline Dureza $\left(\mathrm{kg} / \mathrm{cm}^{2}\right)$ & $0,74^{c}$ & $0,73^{c}$ & $1,10^{\mathrm{b}}$ & $1,73^{\mathrm{a}}$ & 0,07 & $* * *$ \\
\hline \multicolumn{7}{|l|}{ COLOR } \\
\hline $\mathrm{L}^{*}$ & $47,69^{a}$ & $44,14^{b}$ & $45,07^{b}$ & $39,60^{c}$ & 0,49 & $* * *$ \\
\hline$a^{*}$ & $7,98^{\mathrm{b}}$ & $10,09^{a}$ & $7,57^{\mathrm{b}}$ & $10,91^{a}$ & 0,26 & $* * *$ \\
\hline$b^{*}$ & $8,73^{a}$ & $8,09^{a}$ & $7,36^{b}$ & $5,92^{c}$ & 0,18 & $* * *$ \\
\hline
\end{tabular}

PCF= peso canal fría; ${ }^{1}$ Lechazo de Castilla-León. ${ }^{2}$ Ternasco de Aragón. Distintas letras en la misma fila indican diferencias significativas $(\mathrm{p} \leq 0,05)$; e.e.m: error estándar de la media.

(2010), en la raza italiana Apenninica, encontraron diferencias en el peso canal según la época de nacimiento y cría, debido a las variaciones en la alimentación de las ovejas, siendo, igualmente, los lechales de otoño los que presentaron un peso ligeramente superior respecto a los de primavera.

En relación a la composición tisular (tabla I), las diferencias entre lotes fueron significativas para todos los componentes de la disección ( $\mathrm{p} \leq 0,001)$, mostrando el porcentaje de otros componentes una significación menor $(p \leq 0,01)$. Se encontraron diferencias significativas entre todos los tipos de animales, presentando los lechales de la raza Churra Tensina de otoño un menor porcentaje de músculo y hueso, así como un mayor porcentaje de grasa total y subcutánea que los lechales tensinos de primavera o los lechales con IGP. Posiblemente, estas diferencias se encuentren ligadas al sistema de producción-alimentación de las ovejas, ya que, las madres de partos de otoño tuvieron acceso a los abundantes pastos de puerto, además de una posterior suplementación, lo que modificaría las características y disponibilidad de la leche, repercutiendo en una mayor disponibilidad energética de los lechales y en la consiguiente superior deposición de grasa. El estudio de D’Alessandro et al. (2013) reflejó también cómo en función de la época de cría-sacrificio los porcentajes de hueso y de grasa varían, pudiendo verse afectados por las condiciones medioambientales de cada estación, y en consecuencia produciendo una tasa diferente en el crecimiento diferencial de los tejidos. En dicho estudio se señaló que los lechales de primavera (con 45 días de edad) presentaban resultados más favorables en el ratio músculo/hueso que los lechales de invierno de la misma edad. En el presente estudio, entre ninguno de los tres tipos de lechales ovinos hubo diferencias significativas en el ratio músculo/hueso.

En relación a las diferencias entre tipos comerciales, los ternascos presentaron el mayor porcentaje de músculo y menor de hueso, reflejo de la evolución natural de estos tejidos en el desarrollo de los animales, así como de su mayor peso. En comparación con los lechales de la Raza Churra Tensina de sacrificios de otoño, el Ternasco de Aragón presentó una menor cantidad de grasa, debido posiblemente a un descenso relativo de la ingesta de energía de los animales destetados (Geenty et al., 1985). Ambos tipos de lechales de la raza Churra Tensina mostraron un porcentaje de músculo significativamente inferior al de los otros dos tipos de corderos de IGP, lo que a su vez se acompañó de un mayor porcentaje de grasa total y un porcentaje de hueso inferior con respecto al Lechazo de Castilla-León.

\section{Calidad instrumental de la CARNe}

En relación al $\mathrm{pH}$ final, todos los valores se encontraron dentro del rango de $\mathrm{pH}$ normales para este tipo de carne (Martínez Cerezo et al., 2005a), aunque la carne de los lechales Churra Tensina de otoño presentó un valor medio inferior $(p \leq 0,001)$ al del resto de los grupos. La época de sacrificio también tuvo un efecto en otros estudios como los de D’Alessandro et al. (2013), en los que los pHs de la carne de los animales de invierno fueron superiores a los que presentaron la carne de los animales de primavera. También en Mazzone et al. (2010), los lechales de invierno presentaron pHs superiores a los de otoño. Los resultados en la bibliografía referentes al efecto del peso vivo o engrasamiento sobre el $\mathrm{pH}$ son poco homogéneos en lechales, asociándose estas diferencias a variaciones en 
el contenido en glucógeno del músculo, influenciado por el estrés presacrificio y/o diferencias en el sistema de alimentación de las ovejas (Mazzone et al., 2010; D’Alessandro et al., 2013).

En relación a la textura, hubo diferencias significativas $(\mathrm{p} \leq 0,001)$ entre el Ternasco de Aragón y los otros tres tipos comerciales en la carga máxima. Igualmente, en la dureza las diferencias fueron significativas entre ambos productos IGP y ambos lechales de raza Churra Tensina (tabla I), sin observarse, por lo tanto, estos valores afectados por la época de producción de los lechales de Churra Tensina. Así mismo, en los trabajos de D'Alessandro et al. (2013), tampoco se hallaron diferencias en la textura de la carne entre diferentes épocas de sacrificio de los animales que se estudiaron. La fuerza necesaria para cortar las muestras fue superior en los animales de categoría ternasco (tabla I), lo que podría estar asociado a una mayor edad y a la posible presencia de más colágeno insoluble (Lepetit, 2007). Los lechales de raza Churra Tensina, en ambos sistemas de producción, presentaron valores de dureza inferiores a los del Lechazo Castilla-León (IGP), lo que se podría explicar por diferencias en el peso vivo, raza, estado de engrasamiento o potencial de ablandamiento de la carne. Por una parte, varios estudios han comprobado cómo la fuerza de corte aumenta con el peso vivo (Sañudo et al., 1996; Teixeira et al., 2005; Martínez Cerezo et al., 2005a), presentando los animales más ligeros una menor dureza de la carne, al igual que ocurre en este trabajo. Carrasco et al. (2009) en animales de raza Churra Tensina procedentes de diferentes sistemas de alimentación y sacrificados con un mayor peso (22-24 $\mathrm{kg}$ PV) obtuvieron a 4 días de maduración valores superiores $\left(1,05-1,54 \mathrm{~kg} / \mathrm{cm}^{2}=10,3-15,1 \mathrm{~N} / \mathrm{cm}^{2}\right)$ a $\mathrm{los}$

Tabla II. Composición en ácidos grasos (\% ácidos grasos totales) de la grasa intramuscular del músculo Longissimus thoracis lumborum de lechales comerciales de raza Churra Tensina sacrificados en primavera $\mathrm{u}$ otono respecto a dos tipos ovinos con Indicación Geográfica Protegida (IGP) (Fatty acid composition (\% total fatty acids) from intramuscular fat of Longissimus thoracis lumborum muscle of Churra Tensina commercial suckling lambs slaughtered in spring or autumn, compared with two commercial lambs within a Protected Geographical Indication (IGP)).

\begin{tabular}{|c|c|c|c|c|c|c|}
\hline & Churra tensina primavera & Churra tensina otoño & Lechazo' IGP & T.A. $^{2}$ IGP & e.e.m. & Sig. \\
\hline Grasa intramuscular & $1,95^{\mathrm{ab}}$ & $2,15^{a}$ & $1,70^{\mathrm{b}}$ & $1,87^{\mathrm{b}}$ & 0,05 & ** \\
\hline C 10:0 & $0,21^{\mathrm{a}}$ & $0,20^{\mathrm{a}}$ & $0,20^{a}$ & $0,13^{b}$ & 0,01 & $* * *$ \\
\hline C $12: 0$ & $0,50^{\mathrm{a}}$ & $0,38^{\mathrm{b}}$ & $0,48^{a}$ & $0,21^{c}$ & 0,02 & $* * *$ \\
\hline C 14:0 & $4,43^{a}$ & $4,15^{\mathrm{a}}$ & $4,47^{a}$ & $2,64^{b}$ & 0,15 & $* * *$ \\
\hline C $14: 1$ & $0,14^{\mathrm{a}}$ & $0,17^{\mathrm{a}}$ & $0,16^{a}$ & $0,09^{b}$ & 0,01 & $* * *$ \\
\hline C 15:0 & 0,43 & 0,36 & 0,43 & 0,41 & 0,01 & ns \\
\hline C 16:0 & $19,89^{b c}$ & $19,65^{\mathrm{c}}$ & $21,44^{\mathrm{a}}$ & $21,01^{a b}$ & 0,22 & ** \\
\hline C $16: 1$ & 1,80 & 2,03 & 1,95 & 1,84 & 0,05 & ns \\
\hline C 17:0 & $0,98^{b}$ & $1,03^{\mathrm{b}}$ & $0,90^{\mathrm{b}}$ & $1,49^{a}$ & 0,04 & $* * *$ \\
\hline C $17: 1$ & $0,47^{b}$ & $0,55^{\mathrm{b}}$ & $0,48^{b}$ & $0,85^{a}$ & 0,03 & $* * *$ \\
\hline C 18:0 & 13,43 & 12,87 & 12,42 & 12,91 & 0,16 & ns \\
\hline C $18: 1 n-9$ & $30,59^{b}$ & $33,51^{a}$ & $30,01^{b}$ & $33,45^{a}$ & 0,40 & $* * *$ \\
\hline C $18: 2 n-6$ & $7,27^{\mathrm{bc}}$ & $6,89^{c}$ & $8,98^{a}$ & $8,36^{\mathrm{ab}}$ & 0,23 & $* *$ \\
\hline CLA & $1,56^{\mathrm{a}}$ & $1,34^{\mathrm{b}}$ & $0,69^{c}$ & $0,71^{\mathrm{c}}$ & 0,06 & $* * *$ \\
\hline C $18: 3 n-6$ & 0,05 & 0,05 & 0,06 & 0,08 & 0,01 & ns \\
\hline C $18: 3 n-3$ & $1,99^{a}$ & $2,13^{a}$ & $1,31^{b}$ & $0,84^{\circ}$ & 0,11 & $* * *$ \\
\hline C 20:0 & $0,12^{\mathrm{b}}$ & $0,11^{b}$ & $0,13^{a}$ & $0,09^{c}$ & 0,00 & $* * *$ \\
\hline C $20: 1$ & 0,15 & 0,15 & 0,16 & 0,14 & 0,00 & ns \\
\hline C $20: 2 n-6$ & $0,08^{a b}$ & $0,07^{\mathrm{b}}$ & $0,09^{a}$ & $0,07^{b}$ & 0,00 & * \\
\hline C $20: 2 n-3$ & $0,06^{b}$ & $0,05^{c}$ & $0,08^{a}$ & $0,06^{b}$ & 0,00 & $* * *$ \\
\hline C $20: 3 n-6$ & $0,29^{b}$ & $0,25^{\mathrm{b}}$ & $0,40^{\mathrm{a}}$ & $0,28^{b}$ & 0,01 & $* * *$ \\
\hline C $20: 3 n-3$ & $0,31^{a}$ & $0,30^{\mathrm{a}}$ & $0,37^{a}$ & $0,22^{\mathrm{b}}$ & 0,01 & $* * *$ \\
\hline C $20: 4 n-6$ & $3,11^{b}$ & $2,86^{b}$ & $5,01^{a}$ & $3,19^{b}$ & 0,17 & $* * *$ \\
\hline C $20: 5 n-3$ & $2,00^{a}$ & $1,94^{\mathrm{a}}$ & $1,28^{b}$ & $0,78^{c}$ & 0,10 & $* * *$ \\
\hline C 22:0 & $0,66^{b}$ & $0,48^{c}$ & $0,92^{\mathrm{a}}$ & $0,38^{d}$ & 0,03 & $* * *$ \\
\hline C $22: 6 n-3$ & $1,19^{a}$ & $1,11 a$ & $1,12^{\mathrm{a}}$ & $0,42^{\mathrm{b}}$ & 0,06 & $* * *$ \\
\hline$\%$ SAT & $40,65^{\mathrm{ab}}$ & $39,24^{b}$ & $41,40^{\mathrm{a}}$ & $39,28^{b}$ & 0,33 & * \\
\hline$\%$ INSAT & $51,10^{\mathrm{b}}$ & $53,42^{\mathrm{a}}$ & $52,17^{\mathrm{ab}}$ & $51,39^{b}$ & 0,31 & $*$ \\
\hline$\%$ MONO & $33,16^{b}$ & $36,42^{a}$ & $32,76^{b}$ & $36,38^{a}$ & 0,42 & $* * *$ \\
\hline$\%$ POLI & $17,93^{\mathrm{a}}$ & $17,00^{\mathrm{ab}}$ & $19,41^{a}$ & $15,01^{\mathrm{b}}$ & 0,47 & $* *$ \\
\hline$\% n-6$ & $10,81^{b}$ & $10,13^{b}$ & $14,54^{\mathrm{a}}$ & $11,99^{b}$ & 0,26 & $* * *$ \\
\hline$\% n-3$ & $5,56^{a}$ & $5,53^{a}$ & $4,17^{b}$ & $2,32^{c}$ & 0,39 & $* * *$ \\
\hline POLI/SAT & 0,44 & 0,43 & 0,48 & 0,38 & 0,01 & ns \\
\hline$n-6 / n-3$ & $1,96^{c}$ & $1,84^{c}$ & $3,42^{b}$ & $5,17^{a}$ & 0,03 & $* * *$ \\
\hline
\end{tabular}

Distintas letras en la misma fila indican diferencias significativas ( $\leq \leq 0,05) ;{ }^{1}$ Lechazo de Castilla-León; ${ }^{2} T e r n a s c o$ de Aragón; SAT= saturados; INSAT= insaturados; MONO= monoinsaturados; $\mathrm{POLI}=$ poliinsaturados; e.e.m= error estándar de la media. 
del presente estudio (tabla I). Igualmente un mayor engrasamiento se puede asociar a una carne más tierna (Sañudo et al., 2000).

El color de la carne fue significativamente diferente entre los tipos comerciales en todas las variables estudiadas. El Ternasco de Aragón presentó una carne menos luminosa (menor $\left.\mathrm{L}^{*}\right)$, con mayor índice de rojo $\left(\mathrm{a}^{*}\right)$ y menor índice de amarillo $\left(b^{*}\right)$, típica de animales de más edad, destetados y con una alimentación en base a concentrados. Los resultados son similares a los de Martínez Cerezo et al. (2005a) en la raza Rasa Aragonesa, en los que se observa como al aumentar la edad de los corderos, disminuye la luminosidad, aumentando el índice de rojo. Los lechales de raza Churra Tensina de primavera presentaron la carne más luminosa y con menor $\mathrm{a}^{*}$, junto con los lechales con IGP, y mayor $\mathrm{b}^{*}$, junto con el tipo lechal tensinos de otoño. En este sentido, lo esperable sería que los animales no destetados presentasen valores inferiores en el índice de rojo $a^{*}$, debido a una menor presencia de mioglobina asociada a un menor consumo de hierro (Sañudo et al., 1998), así como este índice aumentaría en animales criados a pasto (Martínez-Cerezo et al., 2005a) debido a una mayor concentración de estos pigmentos hemínicos.

\section{PerfiL LiPÍDICO}

La tabla II recoge el porcentaje de grasa intramuscular de la carne de cada grupo de animales, así como el porcentaje de cada ácido graso respecto al total de los ácidos grasos identificados y las relaciones entre los diferentes grupos de ácidos grasos. Los animales de raza Churra Tensina de otoño, que presentaron un mayor porcentaje de grasa (tanto total como subcutánea), fueron los que también presentaron un porcentaje superior de grasa intramuscular $(\mathrm{p} \leq 0,01)$ en relación al Lechazo Castilla-León IGP y al Ternasco de Aragón IGP. Mazzone et al. (2010) encontraron un mayor porcentaje de grasa intramuscular en los lechales criados en otoño con respecto a los de invierno, asociando este efecto a las diferencias en la calidad de la leche materna, siendo ésta más rica en grasa cuando los animales han estado en pastoreo que cuando se han alimentado con concentrado, por el mayor contenido en fibra de la dieta. Sin embargo, las diferencias entre los animales de primavera y otoño no fueron lo suficientemente importantes para modificar, de forma significativa, la cantidad de grasa intramuscular.

La época de producción de los lechales de raza Churra Tensina modificó el perfil de algunos ácidos grasos, de modo que los lechales de primavera presentaron unos porcentajes significativamente superiores de C 12:0, CLA, C 20:2 n-3, y C 22:0 que los de otoño, mientras que los animales de otoño presentaron un porcentaje superior de C 18:1 n-9 que los de primavera. El efecto de la época de producción en el perfil de los ácidos grasos en lechales también fue hallado por Mazzone et al. (2010), sin embargo no existe una total concordancia respecto a los ácidos grasos que varían y la dirección de los cambios. El contenido en ácidos grasos de cadena corta en lechales se encuentra relacionado directamente con el perfil de la leche. Así, se encontró un mayor porcentaje de ácido laúrico C 12:0 en animales cuyas madres pastaban (Mazzone et al., 2010). Igualmente, Valvo et al. (2005) encontraron que el aumento de CLA en la carne se puede obtener no sólo como consecuencia del pastoreo directo, sino también en lechales cuyas madres hayan tenido acceso a pastos. En base a esto, cabría esperar en nuestro estudio un mayor porcentaje de CLA en los animales de otoño, ya que las madres estuvieron pastando, pero sin embargo, fue superior en los de primavera, debido, posiblemente, a que las ovejas recibieron heno durante la estabulación, ya que se ha documentado que existen variaciones en este ácido graso y sus precursores en la leche de las ovejas, debido a los cambios estacionales que presentan los pastos mediterráneos durante las diferentes épocas del año (Park et al., 2007). Por otra parte, tal y como señalaron Biondi et al. (2008), al segundo o tercer día de introducir las ovejas en pastoreo ya se encuentran modificaciones en el perfil de la leche materna, lo que unido a las variaciones en la concentración de ácidos grasos poliinsaturados de las distintas especies forrajeras (Mazzone et al., 2010) podría explicar las variaciones entre los dos grupos de animales de raza Churra Tensina, aunque el periodo de pastoreo haya sido notablemente mayor en las ovejas de otoño. De acuerdo con Joy et al. (2012), la alimentación de las ovejas durante la lactación tiene más influencia sobre el perfil de los ácidos grasos de los corderos que la alimentación durante la gestación. Sin embargo, Joy et al. (2008) al comparar corderos de Churra Tensina (22$24 \mathrm{~kg}$ peso vivo) criados en sistemas de pastoreo o con concentrado, no obtuvieron diferencias significativas en el perfil de los ácidos grasos, lo que podría estar asociado al corto periodo de cría y a la similitud entre ambos grupos del porcentaje de grasa intramuscular obtenido. De manera global, se podría decir que el perfil de los ácidos grasos hallado en el presente estudio para la raza Churra Tensina es similar a los resultados de Panea et al. (2010) realizados sobre lechales de la raza Ansotana, criados en una región y en unas condiciones similares a las del presente estudio, como son las asociadas a los sistemas de montaña en el Pirineo.

Al comparar los cuatro grupos entre sí, se observaron diferencias significativas en todos los ácidos grasos, excepto en C 15:0, C 16:1, C 18:0, C 18:3 n-6 y C 20:1, debidas fundamentalmente a las diferencias en el perfil entre las categorías lechal y ternasco. En general, los ácidos grasos de cadena corta fueron más abundantes en los lechales, debido a su alimentación láctea, al ser la grasa de la leche especialmente rica en este tipo de ácidos grasos (Valvo et al., 2003), y a la ausencia de actividad ruminal, lo que permite una deposición directa de la grasa ingerida. El ácido graso C 17:0, cuyas variaciones han sido asociadas a una mayor ingesta de concentrando, fue superior en el ternasco (Mazzone et al., 2010). Tal y como ha sido descrito en otros trabajos, las dietas forrajeras aumentan la presencia de CLA y n-3 en la leche de las madres (Valvo et al., 2003), lo que al igual que en otros estudios (Ripoll-Bosch et al., 2012) repercutió en la composición de la carne de lechal. En términos generales, ambos tipos de lechales de la raza Churra Tensina presentaron unos porcentajes de CLA, $n$-3 y $\alpha$ - linolénico (C 18:3n-3) significativamente superiores a los del Lechazo Castilla-León (IGP) y del Ternasco de Aragón, grupos en los que la alimentación de las madres (lechal) o del propio animal (ternasco) 
Tabla III. Calidad sensorial del músculo Longissimus thoracis lumborum evaluadas por un panel entrenado en lechales comerciales de raza Churra Tensina sacrificados en primavera u otoño respecto a dos tipos ovinos con Indicación Geográfica Protegida (IGP) (Sensory quality of the Longissimus thoracis lumborum muscle evaluated by a trained panel from Churra Tensina commercial suckling lambs slaughtered in spring or autumn, compared with two commercial lambs within a Protected Geographical Indication (IGP)).

\begin{tabular}{lcccccc}
\hline & Churra tensina primavera & Churra tensina otoño & Lechazo ${ }^{1}$ IGP & T.A. $^{2}$ IGP & e.e.m. & Sig. \\
\hline Olor a cordero & $4,50^{\mathrm{b}}$ & $4,60^{\mathrm{b}}$ & $4,64^{\mathrm{ab}}$ & $5,01^{\mathrm{a}}$ & 0,07 & $*$ \\
Olor a grasa & 2,61 & 2,26 & 2,41 & 2,35 & 0,07 & $\mathrm{~ns}$ \\
Olor a lácteo & 1,25 & 1,26 & 1,18 & 1,20 & 0,05 & $\mathrm{~ns}$ \\
Terneza & $6,92^{\mathrm{a}}$ & $6,86^{\mathrm{a}}$ & $5,89^{\mathrm{b}}$ & $4,39^{\mathrm{c}}$ & 0,18 & $* * *$ \\
Jugosidad & $4,74^{\mathrm{a}}$ & $4,82^{\mathrm{a}}$ & $4,56^{\mathrm{a}}$ & $4,21^{\mathrm{b}}$ & 0,07 & $* *$ \\
Fibrosidad & $2,88^{\mathrm{c}}$ & $2,98^{\mathrm{c}}$ & $3,77^{\mathrm{b}}$ & $5,02^{\mathrm{a}}$ & 0,15 & $* * *$ \\
Flavor a cordero & 5,47 & 5,34 & 5,34 & 5,45 & 0,05 & $\mathrm{~ns}$ \\
Flavor a grasa & 3,83 & 3,91 & 3,95 & 3,71 & 0,06 & $\mathrm{~ns}$ \\
Flavor a lácteo & 1,41 & 1,43 & 1,42 & 1,48 & 0,05 & $\mathrm{~ns}$ \\
Flavor a metálico & 3,21 & 3,26 & 3,33 & 3,67 & 0,07 & $\mathrm{~ns}$ \\
\hline
\end{tabular}

Distintas letras en la misma fila indican diferencias significativas ( $\leq \leq 0,05) ;{ }^{1}$ Lechazo de Castilla-León; ${ }^{2}$ Ternasco de Aragón; e.e.m= error estándar de la media.

pudo ser más concentrada. El porcentaje de ácidos grasos monoinsaturados e insaturados totales, varió entre los dos grupos de lechales Churra Tensina. Por otro lado, los lechales de dicha raza presentaron un menor porcentaje en ácidos $n-6$ y mayor proporción de $n$-3 respecto a los animales de ambas IGPs, lo que les confiere un ratio $n-6 / n-3$ menor, resultado probablemente justificado por una alimentación de las madres más rica en forraje (pasto y heno), como ya se ha comentado previamente.

\section{CALIDAD SENSORIAL}

En la tabla III se recogen los resultados sensoriales obtenidos a partir del panel entrenado. Entre los atributos evaluados hubo diferencias significativas en terneza y fibrosidad ( $p \leq 0,001)$, así como en la jugosidad $(\mathrm{p} \leq 0,01)$ e intensidad de olor a cordero $(\mathrm{p} \leq 0,05)$.

La carne procedente de ambos tipos comerciales de la raza Churra Tensina presentó las mayores puntuaciones en terneza y las menores en fibrosidad, sin diferencias entre las épocas de producción de los lechales. El Ternasco de Aragón fue el grupo que obtuvo las puntuaciones más bajas en terneza, acompañadas de las más altas en fibrosidad, encontrándose el Lechazo de Castilla-León en posiciones intermedias. Estos resultados coinciden con los valores obtenidos en la determinación instrumental de la dureza (tabla I). Coincidiendo con Martínez Cerezo et al. (2005b), los animales tipo lechal (Churra Tensina y Churra) obtu- vieron una mayor jugosidad respecto al tipo ternasco (Rasa Aragonesa). En cambio, como era de esperar, el olor a cordero aumentó con el peso-edad y, así los ternascos tuvieron mayores intensidades que los tipos tensinos, presentando el lechazo de Castilla-León IGP unos valores intermedios sin diferencias significativas con los otros grupos.

Los atributos relacionados con la intensidad de olor y flavor a grasa y lácteo presentaron en todos los grupos unos valores bajos, inferiores a 4 puntos en una escala de 10, así como el flavor metálico, al igual que ocurrió en los estudios de Panea et al. (2010) con lechales de las razas Ansotana y Rasa Aragonesa. La ausencia de diferencias en las diferentes notas de flavor podría deberse a la juventud de los animales utilizados, ya que de forma global el flavor es poco notable a edades tempranas en ovino y aumenta con la edad (Martínez Cerezo et al., 2005b).

\section{CONSUMIDORES}

En la tabla IV se puede observar cómo la aceptabilidad de los tres atributos valorados (aceptabilidad de la terneza, del sabor y aceptabilidad general) fue significativamente diferente $(p \leq 0,001)$ entre los tipos comerciales, siendo ambos tipos de lechales de la raza Churra Tensina los que obtuvieron una mayor puntuación, mostrando los lechazos de Castilla-León (IGP) una aceptabilidad intermedia con respecto a los demás grupos. Por otra parte, los resultados muestran cómo, a

Tabla IV. Aceptabilidad de lechales comerciales de raza Churra Tensina sacrificados en primavera u otoño respecto a dos tipos ovinos con Indicación Geográfica Protegida (IGP), evaluado por consumidores en condiciones estandarizadas $(n=120)^{2}$ (Acceptability of Churra Tensina commercial suckling lambs slaughtered in spring or autumn compared with two commercial lambs within a Protected Geographical Indication (IGP), evaluated by consumers under standardized conditions $\left.(n=120)^{x}\right)$.

\begin{tabular}{lcccccc}
\hline & Churra tensina primavera & Churra tensina otoño & Lechazo ${ }^{1}$ IGP & T.A. ${ }^{2}$ IGP & e.e.m. & Sig. \\
\hline Aceptabilidad general & $7,23^{\mathrm{a}}$ & $7,18^{\mathrm{a}}$ & $6,29^{\mathrm{b}}$ & $5,65^{\mathrm{c}}$ & 0,79 & $* * *$ \\
Aceptabilidad terneza & $7,58^{\mathrm{a}}$ & $7,58^{\mathrm{a}}$ & $6,14^{\mathrm{b}}$ & $4,98^{\mathrm{c}}$ & 0,88 & $* * *$ \\
Aceptabilidad sabor & $7,08^{\mathrm{a}}$ & $7,13^{\mathrm{a}}$ & $6,39^{\mathrm{b}}$ & $6,15^{\mathrm{b}}$ & 0,72 & $* * *$ \\
\hline
\end{tabular}

'Escala de aceptación de 9 puntos (1= me desagrada extremadamente/9= me gusta extremadamente); ${ }^{1}$ Lechazo de Castilla-León; ${ }^{2}$ Ternasco de Aragón; Distintas letras en la misma fila indican diferencias significativas $(p \leq 0,05)$; e.e.m= error estándar de la media. 
pesar de existir diferencias en el contenido y en la calidad de la grasa entre las dos épocas de producción de los lechales de la raza Churra Tensina, el consumidor no percibe el producto como diferente, presentando una buena aceptabilidad ambas épocas de producción.

En general, la aceptabilidad de los diferentes grupos, para todos los parámetros analizados, se podría considerar que fue buena, obteniéndose valoraciones superiores al 5,5 sobre una escala hedónica de 9 puntos. Sólo la terneza del Ternasco de Aragón IGP fue puntuada ligeramente más baja, pero superando los 4,5 puntos, lo que se correspondería con una nota aceptable. Esta nota inferior se podría deber a que los animales del tipo Ternasco de Aragón IGP fueron los que mayor dureza instrumental y menor terneza sensorial mostraron. En general, los consumidores prefieren la carne procedente de ovinos ligeros con maduraciones intermedias (a partir de 4 días) (Martínez-Cerezo et al., 2005c).

\section{CONCLUSIONES}

Los resultados obtenidos sugieren una buena aceptación del del cordero lechal obtenido a partir de la raza Churra Tensina, independientemente de su época de producción, lo que reflejaría su potencial para la diversificación con éxito del producto obtenido, mayoritariamente corderos de tipo ligero, lo que ayudaría a mejorar la renta de los productores y a la consolidación de la raza. Las diferencias entre los tipos de lechales tensinos obtenidos en primavera o en otoño estuvieron relacionadas fundamentalmente con la cantidad y calidad de la composición en ácidos grasos de la grasa. Sin embargo, estas diferencias no fueron lo suficientemente importantes para ser percibidas a nivel sensorial, ya sea a través de la valoración objetiva de un panel entrenado o por medio de estudios de consumidores. Por otro lado, al comparar dichos productos con otros de referencia en el mercado (Lechazo de Castilla-León IGP y Ternasco de Aragón IGP), se observaron diferencias significativas en la composición tisular de la canal, calidad instrumental de la carne, composición de la grasa intramuscular, calidad sensorial y aceptabilidad de la carne, lo que justificaría un posible nicho de mercado específico para estos productos tensinos.

\section{AGRADECIMIENTOS}

A la Asociación de Criadores de Ganado Ovino de Raza Churra Tensina (ATURA) por la financiación, cría y selección de los animales. A Ricardo Azón y a Patricia Lara por su colaboración técnica.

\section{BIBLIOGRAFÍA}

BOE. 2011 . Resolución de 27 de mayo de 2011, de la Dirección General de Industria y Mercados Alimentarios, por la que se publica la modificación del pliego de condiciones de la Indicación Geográfica Protegida Lechazo de Castilla y León. BOE № 152, Sec.III, 68642-68646.

Biondi, L.; Valvo, M.A.; Di Gloria, M.; Scinardo Tenghi, E.; Galorafo, V. and Priolo, A. 2008. Change in ewe milk fatty acids following turning out to pasture. Small Ruminant Res, 75: 17-23.

Bligh, E.G. and Dyer, W.J. 1959. A rapid method of total lipid extraction and purification. Can J Biochem Physiol, 37: 911-917.
Calvo, J.H.; Álvarez-Rodríguez, J.; Marcos-Carcavilla, A.; Serrano, M. and Sanz, A. 2011. Genetic diversity in the Churra tensina and Churra lebrijana endangered Spanish sheep breeds and relationship with other Churra group breeds and Spanish mouflon. Small Ruminant Res, 95: 34-39.

Campo, M.M.; Santolaria, P.; Sañudo, C.; Lepetit, J.; Olleta, J.L.; Panea, B. and Alberti, P. 2000. Assessment of breed type and ageing time effects on beef meat quality using two different texture devices. Meat Sci, 55: 371-378.

Carrasco, S.; Panea, B.; Ripoll, G.; Sanz, A. and Joy, M. 2009. Influence of feeding systems on cortisol levels, fat colour and instrumental meat quality in light lambs. Meat Sci, 83: 50-56.

Carrillho, M.C.; López, M. and Campo, M.M. 2009. Effect of the fattening diet on the development of the fatty acid profile in rabbits from weaning. Meat Sci, 83: 85-95.

CIE. 1976. Commission Internationale de l'Eclairage, $18^{\text {th }}$ session. London, UK. September 1975. CIE publication, 36.

Colomer, F.; Morand Fehr, P.; Kirton, A.H.; Delfa, R. and Sierra, I. 1988. Métodos normalizados para el estudio de los caracteres cuantitativos $y$ cualitativos de las canales caprina y ovinas. Cuadernos INIA: $17.41 \mathrm{pp}$.

D'Alessandro, A.G.; Maiorano, G.; Ragni, M.; Casamassima, D.; Marsico, G. and Martemucci, G. 2013. Effects of age and season of slaughter on meat production of light lambs: Carcass characteristics and meat quality of Leccese breed. Small Ruminant Res, 114: 97-104.

Font i Furnols, M.; Gispert, M.; Guerrero, L.; Velarde, A.; Tibau, J.; Soler, J.; Hortós, M.; García-Regueiro, J.A.; Pérez, J.; Suárez, P. and Oliver, M.A. 2008. Consumer's sensory acceptability of pork from immunocastrated male pigs. Meat Sci, 80: 1013-1018.

Geenty, K.G.; Clarke, J.N. ans Wright, D.E. 1985. Lactation performance, growth, and carcass composition of sheep. 2. Relationships between ewe milk production, lamb water turnover, and lamb growth in Rommey, Dorset and crossbred sheep. New Zeal J Agr Res, 28: 249-255.

Joy, M.; Ripoll, G. and Delfa, R. 2008. Effects of feeding system on carcass and non-carcass composition of Churra Tensina light lambs. Small Ruminant Res, 78: 123-133.

Joy, M.; Ripoll, G.; Molino, F.; Dervishi, E. and Alvarez-Rodriguez, J. 2012. Influence of the type of forage supplied to ewes in pre- and post-partum periods on the meat fatty acids of suckling lambs. Meat Sci, 90: 775-782.

Lepetit, J. 2007. A theoretical approach of the relationships between collagen content, collagen cross-links and meat tenderness. Meat Sci, 76: 147-159.

Macfie, H.J.; Bratchell, N.; Greenhoff, K. and Vallis, L.V. 1989. Designs to balance the effect of order presentation and first-order and carry over effects in hall test. J Sens Stud, 4: 129-148.

MAGRAMA. 2011 . Indicación Geográfica Protegida Ternasco de Aragón. Pliego de condiciones. www.magrama.gob.es/es/alimentacion/ temas/calidad-agroalimentaria/calidad-diferenciada/dop/carnes/ dop_ternasco_aragon.aspx. (24/11/2014).

Martínez-Cerezo, S.; Sañudo, C.; Panea, B.; Medel, I.; Delfa, R.; Sierra, I.; Beltrán, J.Á.; Cepero, R. and Olleta, J.L. 2005a. Breed, slaughter weight and ageing time effects on physico-chemical characteristics of lamb meat. Meat Sci, 69: 325-333.

Martínez-Cerezo, S.; Sañudo, C.; Medel, I. and Olleta, J.L. 2005b. Breed, slaughter weight and ageing time effects on sensory characteristics of lamb. Meat Sci, 69: 571-578.

Martínez-Cerezo, S.; Sañudo, C.; Panea, B. and Olleta, J.L. 2005c. Breed, slaughter weight and ageing time effects on consumer appraisal of three muscles of lamb. Meat Sci, 69: 797-805.

Mazzone, G.; Giammarco, M.; Vignola, G.; Sardi, L. and Lambertini, L. 2010. Effects of the rearing season on carcass and meat quality of suckling Apennine light lambs. Meat Sci, 86: 474-478.

Olleta, J.L.; Sañudo, C. and Sierra, I. 1992. Producción de carne en la agrupación ovina Churra tensina: calidad de la canal y de la carne en los tipos ternasco y cordero de cebo. Arch Zootec, 41: 197-208. 
Panea, B.; Joy, M.; Ripoll, G.; Boscolo, J. and Albertí, P. 2010. Características de la canal y de la carne del lechal de raza Ansotana: efecto del sexo. ITEA, 106: 229-244.

Panea, B.; Ripoll, G. and Joy, M. 2013. Caracterización y agrupamiento de algunos tipos comerciales de cordero por su perfil sensorial. ITEA, 109: 303-318.

Park, Y.W.; Juárez, M.; Ramos, M. and Haenlein, G.F.W. 2007. Physicochemical characteristics of goat and sheep milk. Small Ruminant Res, 68: 88-113

Ripoll-Bosch, R.; Ripoll, G.; Álvarez-Rodríguez, J.; Blasco, I.; Panea, B. and Joy, M. 2012. Efecto del sexo y la explotación sobre la calidad de la canal y de la carne del cordero lechal de raza Ojinegra. ITEA, 108: 552-536.

Sanz, A.; Álvarez-Rodríguez, J.; Carcarosa, L.; Ripoll, G.; Carrasco, S.; Revilla, R. and Joy, M. 2008. Características de la canal de los tipos comerciales de cordero lechal, ternasco y pastenco en la raza Churra Tensina. ITEA, 104: 42-57.

Sañudo, C.; Santolaria, M.P.; María, G.; Osorio, M. and Sierra, I. 1996. Influence of carcass weight on instrumental and sensory lamb meat quality in intensive production systems. Meat Sci, 42: 195-202.
Sañudo, C.; Sánchez, A. and Alfonso, M. 1998. Small ruminant production systems and factors affecting meat lamb quality. Meat Sci, 49 (Suppl. 1): S29-S64.

Sañudo, C.; Alfonso, M.; Sánchez, A.; Delfa, R. and Teixeira, A. 2000. Carcass and meat quality in light lambs from different fat classes in the EU carcass classification system. Meat Sci, 56: 89-94.

Teixeira, A.; Batista, S.; Delfa, R. and Cadavez, V. 2005. Lamb meat quality of two breeds with protected origin designation. Influence of breed, sex and live weight. Meat Sci, 71: 530-536.

Valvo, M.A.; Lanza, M.; Bella, M.; Fasone, V.; Scerra, M.; Biondi, L. and Priolo, A. 2005. Effect of ewe feeding system (grass v. concentrate) on intramuscular fatty acids of lambs raised exclusively on maternal milk. Anim Sci, 81: 431-436.

UNE-EN ISO 8589. 2010. Análisis sensorial. Guía general para el diseño de salas de catas.

UNE-EN ISO 8586-2. 2009. Análisis sensorial. Guía general para la selección, entrenamiento y control de evaluadores. 
